\title{
Registro de nado y evidencias indirectas del peludo patagónico Chaetophractus villosus (Desmarest, 1804) en Aysén, Chile
}

\author{
Swimming record and indirect evidence of large hairy armadillo \\ Chaetophractus villosus (Desmarest, 1804) in Aysén, Chile
}

Jorge Mella-Romero ${ }^{\bowtie}$, Jorge Mella Ávila² , Felipe Reyes Cortés ${ }^{2} \&$ Cristián Muñoz Villouta $^{3}$

El peludo o peludo patagónico, Chaetophractus villosus (Desmarest, 1804), es una de las tres especies de armadillos (Cingulata: Chlamyphoridae) existentes en Chile, y presenta una amplia distribución geográfica, ya que además se encuentra en Bolivia, Paraguay y Argentina (Abba et al. 2007; Poljak et al. 2007; Muñoz-Pedreros, 2008). En Chile, esta especie habita en las zonas andinas desde la región del Biobío hasta la región de Magallanes (Tamayo, 2009; Superina \& Pasutti, 2016), y se ha confirmado su presencia como especie introducida en el territorio chileno de Isla Grande de Tierra del Fuego (Cabello et al. 2017). C. villosus es una especie de la cual se han publicado artículos sobre fisiología, anatomía, genética y conducta (Casanave et al. 2005; Poljak et al. 2010). A pesar de que está descrito que los miembros de la familia Chlamyphoridae pueden nadar, esto se considera como un comportamiento poco frecuente $y$ asociado a dispersión o escape (véase Talmage \& Buchanan, 1954; Hass et al. 2003; Bender \& Bender, 2013). Hasta la fecha, registros de nado no han sido documentados en C. villosus. De la misma forma, la información sobre evidencias indirectas de armadillos en Chile es escasa, con pocos trabajos publicados que incluyen esta temática (e.g., Muñoz-Pedreros \& Gil, 2009; Tamayo, 2009; Superina \& Pasutti, 2016). El objetivo de este artículo fue describir el registro de conducta natatoria en $C$. villosus $y$ sus evidencias indirectas (e.g., huellas, fecas y madrigueras), en el transcurso de una investigación de campo, realizada en la región de Aysén, Chile.
Durante una prospección terrestre diurna efectuada el día 1 de febrero de 2019, observamos un ejemplar adulto de peludo patagónico (Chaetophractus villosus) a $2,5 \mathrm{~km}$ al norte de Balmaceda (4553'11" S; 7141'56" O, 498 $\mathrm{msnm})$. El individuo fue registrado en ambiente de estepa patagónica. Ante nuestra presencia, el armadillo se movilizó rápidamente hacia el borde del estero Blanco Chico, el que atravesó nadando (Fig. 1), para luego esconderse entre la vegetación arbustiva. El estero es semicorrentoso, de aguas claras, con un ancho estimado de 15 a 20 metros y una profundidad aproximada de 50 a $70 \mathrm{~cm}$ (en la zona de un vado, que pasa por la ruta X-695).

En el borde del estero, el ejemplar dejó su impronta, la que se caracteriza por mostrar básicamente las marcas de las uñas, siendo muy poco evidentes las huellas de los dedos y de los cojinetes plantares (Fig. 2a). Las manos tienen cuatro dedos, pero los extremos están reducidos,

\footnotetext{
1 Laboratorio de Conservación Biológica,

Departamento de Ciencias Ecológicas,

Facultad de Ciencias, Universidad de Chile,

Las Palmeras 3425, Casilla 653, Santiago, Chile.

$\bigotimes$ jmellaromero@gmail.com

2 Cedrem Consultores,

Padre Mariano 82, oficina 1003. Santiago, Chile. jorge.mella@cedrem.cl, felipe.reyes@cedrem.cl

3 Consultora Cristián Muñoz Villouta,

Marchant Pereira 2950, dpto. 906, Santiago, Chile.

cristian.cmunoz@gmail.com
} 

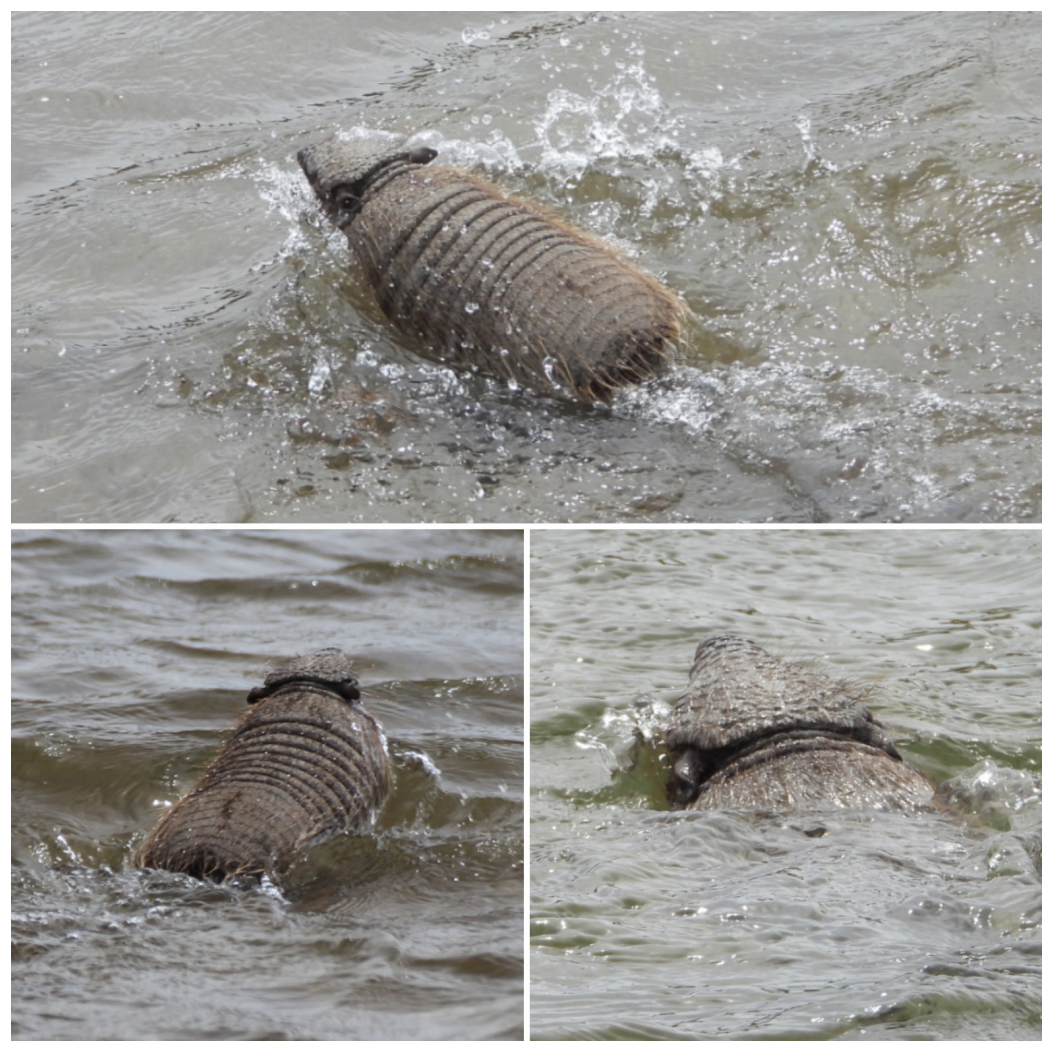

Fig. 1. Secuencia de nado de Chaetophractus villosus en estero Blanco Chico, Balmaceda, región de Aysén.

por lo que pueden observarse dos o tres huellas de uñas centrales (Fig. 2b), siendo la marca de la uña del dedo medio, la más evidente (grande y profunda). Para las mediciones, se utilizó pie de metro, con precisión de 0,1 $\mathrm{mm}$. Las dimensiones de la mano (considerando solo las huellas de las uñas, sin los cojinetes plantares) son de $1,0 \mathrm{~cm}$ de largo y 2,3 $\mathrm{cm}$ de ancho. En las extremidades posteriores (con cinco dedos), también los extremos están reducidos, por lo que se ven tres a cuatro uñas, con las dos centrales más juntas entre sí (situadas a la misma altura). La uña del dedo exterior se ubica levemente más atrás, y la uña del dedo interior es más pequeña y está ubicada aún más atrás (Fig. 2c). El largo de la pata (solo uñas) es de $2,4 \mathrm{~cm}$ y su ancho es de $2,6 \mathrm{~cm}$. La distancia entre las huellas de manos y patas (largo del patrón) es de $25 \mathrm{~cm}$. Antes de percatarse de nuestra presencia, el ejemplar defecó en el sustrato. La feca era de color negro brillante, de $2,4 \mathrm{~cm}$ de ancho máximo y $6 \mathrm{~cm}$ de largo, de textura irregular (formada por lóbulos), con un borde convexo y el otro extremo aguzado (Fig. 3a).
En otros sectores recorridos en la región durante las campañas de terreno (específicamente, en las cercanías de Chile Chico), registramos cuevas ( $\mathrm{n}=5$ ) de armadillo, cerca o en la base de arbustos, como Azorella prolifera (neneo) y Colliguaja integerrima (colliguay). Las entradas de las cuevas eran semicirculares, cavadas en el sustrato arenoso y/o terroso (Fig. 3b), y las dimensiones de estas madrigueras eran de 14 a $16 \mathrm{~cm}$ de largo y 7 a $9 \mathrm{~cm}$ de alto. Además de la distribución geográfica coincidente, el ejemplar observado fue adscrito a un individuo adulto de C. villosus por el tamaño estimado, cercano a los $40 \mathrm{~cm}$. Tamayo (2009) menciona que la longitud total de esta especie alcanza los $48 \mathrm{~cm}$, siendo la mayor de las tres especies de armadillos presentes en Chile, ya que el quirquincho de la puna (Chaetophractus vellerosus) alcanza los 40 $\mathrm{cm}$, y el piche (Zaedyus pichiy), los $34 \mathrm{~cm}$ (Wetzel et al. 2007; Muñoz-Pedreros, 2008).

Existen artículos que informan y describen registros de armadillos nadando, pero estos son 
casi exclusivamente para las especies del hemisferio norte (véase e.g., Talmage \& Buchanan, 1954; Hass et al. 2003; Bender \& Bender, 2013). Para este grupo de vertebrados, la actividad natatoria significa una gran cantidad de gasto energético, dado el peso de las placas óseas de la coraza (i.e., baja flotabilidad; Talmage \& Buchanan, 1954). Por lo anterior, no es extraño que sea un fenómeno poco frecuente y que ocurra excepcionalmente, sugiriéndose como método de escape ante un depredador, o como medio de traslado obligado para movilizarse de una orilla a otra (Talmage \& Buchanan, 1954; Hass et al. 2003). En el caso del registro que se detalla en este artículo, al percatarse de nuestra presencia a unos pocos metros, el ejemplar de C. villosus realizó dicha conducta muy probablemente a modo de escape.

Las huellas de C. villosus registradas en la arena, no son tan evidentes como las de otros mamíferos de mediano tamaño presentes en el área (como mustélidos y lagomorfos), y solo pudimos observar las uñas. Lo anterior concuerda en parte con lo detallado por Muñoz-Pedreros (2008), quien indica un tamaño de la mano de $4,5 \mathrm{~cm}$ de largo y $3,0 \mathrm{~cm}$ de ancho (aunque dichas medidas parecen incluir los cojinetes plantares, lo que no se pudo observar en este registro, por lo que las dimensiones medidas por nosotros son menores). En un sustrato de arena más fina y blanda probablemente se puedan registrar los cojinetes plantares y dedos. En general, en la literatura existente sobre armadillos sudamericanos, pareciera que las especies de este grupo no se pueden reconocer fácilmente por sus improntas (véase Muñoz-Pedreros, 2008), por lo que este artículo complementa la escasa información documentada a la fecha en C. villosus; como el tipo y largo del patrón (distancia entre extremidades superiores e inferiores), de $25 \mathrm{~cm}$.

Nosotros asignamos las cuevas observadas en el sector de Chile Chico a C. villosus, por el tamaño y la forma semicircular de la entrada de las madrigueras, lo que las distingue claramente de las pertenecientes a otros mamíferos en la zona. En el sector, habitan conejos (Oryctolagus cuniculus) y liebres (Lepus europaeus), cuyas madrigueras son

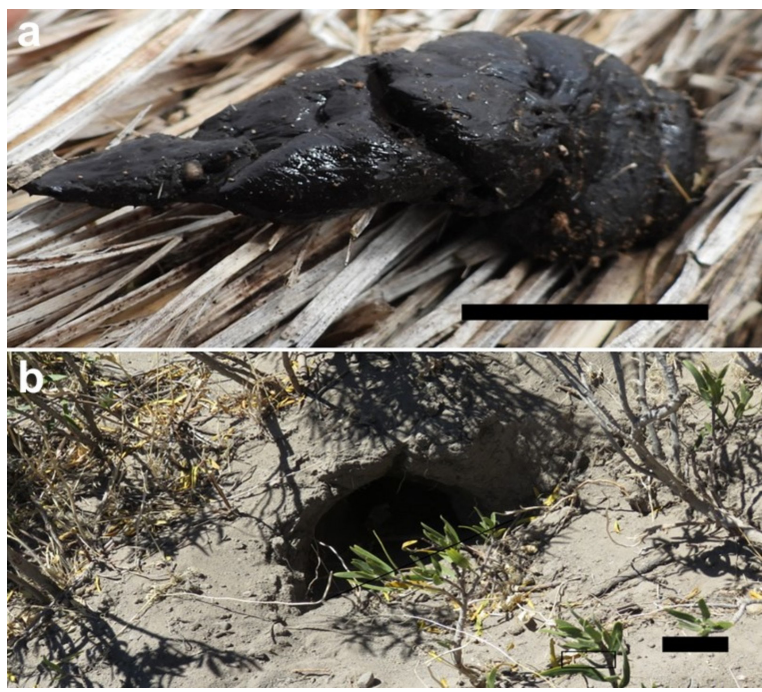

Fig. 3. (a) Feca; (b) madriguera de Chaetophractus villosus (Barras $=2 \mathrm{~cm}$ ).

más grandes, no semicirculares y generalmente con fecas redondas en la entrada (Muñoz-Pedreros, 2008). Otro mamífero presente en el área y que construye madrigueras es el tuco tuco de Coyhaique (Ctenomys coyhaiquensis), roedor fosorial, de pequeño tamaño, cuyas madrigueras son generalmente circulares (como las de todos los Ctenomys), y de un tamaño significativamente 
menor que las de C. villosus (Muñoz-Pedreros \& Gil, 2009). La adscripción de las cuevas a $C$. villosus, se basa además en la similitud en forma y tamaño con las de su congénere $C$. vellerosus, el quirquincho de la puna (presente en el extremo norte del país), cuyas cuevas también tienen forma de arco, con un diámetro máximo de $16 \mathrm{~cm}$ (Zúñiga \& Araya, 2014), concordante con nuestros registros. Las dimensiones de las cuevas registradas son muy similares a las medidas de un cadáver de un ejemplar de C. villosus (hallado en Chile Chico), cuyo diámetro era de $16 \mathrm{~cm}$ y su altura de $9 \mathrm{~cm}$. Este artículo aporta un registro novedoso de un antecedente conductual en $C$. villosus, además de una descripción detallada de evidencias indirectas de esta especie de armadillo. Finalmente, enfatizamos la necesidad de realizar muestreos exhaustivos en la estepa patagónica, dado que en Chile es una zona biogeográfica extensa, pero poco conocida, con escasos trabajos actuales sobre fauna nativa $y$ amenazada (Mella et al. 2018, 2019).

\section{AGRADECIMIENTOS}

JMR agradece a la Comisión Nacional de Investigación Científica y Tecnológica, Beca de Doctorado Nacional/2019-21190472. JMA, FRC y CMV agradecen a Cedrem Consultores y a la Consultora Cristián Muñoz Villouta la oportunidad de redactar esta publicación. Finalmente, agradecemos a los revisores anónimos por sus valiosos comentarios.

\section{LITERATURA CITADA}

Abba, A. M., Vizcaíno, S. F., \& Cassini, M. G. (2007). Effects of land use on the distribution of three species of armadillos in the Argentinean Pampas. Journal of Mammology, 88, 502-507.

Bender, R., \& Bender, N. (2013). Brief communication: Swimming and diving behavior in apes (Pan troglodytes and Pongo pygmaeus): First documented report. American Journal of Physical Anthropology, 152(1), 156-162.

Cabello, J. L., Valenzuela, A. E. J., \& Anderson, C. B. (2017). Avance en el proceso de la invasión biológica del peludo
Chaetophractus villosus (Dasypodidae) en la Isla Grande de Tierra del Fuego: un nuevo desafío binacional. Anales del Instituto de la Patagonia, 45(2), 109-115.

Casanave, E. B., Bermúdez, P. M., \& Polini, N. N. (2005). Haemostatic mechanisms of the armadillo Chaetophractus villosus (Xenarthra, Dasypodidae). Comparative Clinical Pathology, 13(4), 171-175.

Hass, A., Rodrigues, F. H. G., \& Oliveira, T. G. (2003). The Yellow Armadillo, Euphractus sexcinctus, in the North/Northeastern Brazilian Coast. Edentata, 5, 46-47.

Mella, J., Mella-Romero, J., Reyes, F., \& Muñoz, C. (2018). Validación de la presencia de Liolaemus kingii (Bell, 1843) (Iguania: Liolaemidae) en Chile. Boletín del Museo Nacional de Historia Natural, 67(2), 137144.

Mella, J., Reyes F., Mella-Romero J., \& Muñoz, C. (2019). Ampliación de distribución geográfica de Diplolaemus darwinii Bell, 1843 (Iguania: Leiosauridae) en Chile: registro en la región de Aysén. Boletín del Museo Nacional de Historia Natural, 68(1), 33-40.

Muñoz-Pedreros, A. (2008). Huellas y signos de mamíferos de Chile ( $1^{\mathrm{a}}$ ed.). Valdivia: CEA Ediciones.

Muñoz-Pedreros, A., \& Gil, C. (2009). Orden Rodentia. En A. Muñoz-Pedreros \& J. Yáñez (Eds.), Mamíferos de Chile (pp. 93-157). Valdivia: CEA Ediciones.

Poljak, S., Escobar, J., Deferrari, G., \& Lizarralde, M. (2007). Un nuevo mamífero en la Tierra del Fuego: el "peludo" Chaetophractus villosus (Mammalia, Dasypodidae) en Isla Grande. Revista Chilena de Historia Natural, 80, 285-294.

Poljak, S., Confalonieri, V., Fasanella, M., Gabrielli, M., \& Lizarralde, M. (2010). Phylogeography of the armadillo Chaetophractus villosus (Dasypodidae Xenarthra): post-glacial range expansion from Pampas to Patagonia (Argentina). Molecular Phylogenetics and Evolution, 55(1), 38-46.

Superina, M., \& Pasutti, R. (2016). Guía de Identificación de las tres especies de Armadillos Presentes en Chile. Santiago de Chile: Armadillos de Chile Ediciones. 
Talmage, R. V., \& Buchanan, G. D. (1954). The armadillo: a review of its natural history, ecology, anatomy and reproductive physiology. Rice Institute Pamphlet, 41, $1-135$. Tamayo, M. (2009). Orden Xenarthra. En
A. Muñoz-Pedreros \& J. Yáñez (Eds.), Mamíferos de Chile (pp. 85-91). Valdivia: CEA Ediciones.

Wetzel, R. M., Gardner, A. L., Redford, K. H., \&
Eisenberg, J. F. (2007). Orden Cingulata Illiger, 1811. In A. L. Gardner (Ed.), Mammals of South America (pp. 128157). Chicago: The University of Chicago Press.

Zúñiga, E., \& Araya, L. (2014). Abundancia relativa y patrones de actividad de Chaetophractus nationi (Thomas, 1894), en la Reserva de la Biósfera Lauca. Biodiversidata, 2, 35-38. 
